\title{
APPEARANCE AND LOCALIZATION OF ACETYLCHOLINESTERASE IN EMBRYOS OF THE LEECH HELOBDELLA TRISERIALIS ${ }^{1}$
}

\author{
SANDRA FITZPATRICK-MCELLIGOTT ${ }^{2}$ AND GUNTHER S. STENT
}

Department of Molecular Biology, University of California, Berkeley, California 94720

\begin{abstract}
The appearance and localization of acetylcholinesterase (AchE) were studied during embryonic development of the leech Helobdella lriserialis. Use of a histochemical stain showed that AchE is present in the polar plasms of the uncleaved leech egg. In the course of the first two cleavages, the Ach $E$ is transmitted along with the polar plasms mainly to the $\mathrm{D}$ blastomere, precursor of mesoderm and ectoderm. At these early developmental stages, AchE also lines the cleavage furrows between the blastomeres. At later stages, AchE is present in the parental meso- and ectoteloblasts and their daughter stem cells that form the germinal bands. At the completion of germinal band coalescence, AchE is present in the rostral and caudal regions of the germinal plate. Thus, the presence of AchE in the early phase of embryogenesis precedes the formation of any nervous tissue. In the late phase of embryogenesis, once the embryo has reached the stage of body closure and a nervous system is present, the distribution of $\mathrm{AchE}$ is that characteristic of the adult leech: AchE is localized in the neuropil of the segmental ganglia of the ventral nerve cord and in the musculature of the body wall. Therefore, at this still quite immature stage, the embryonic nervous system has taken on not only the morphological but also some of the neurochemical characteristics of the adult leech.
\end{abstract}

Acetylcholinesterase (AchE), the enzyme that hydrolyzes the neurotransmitter acetylcholine (Ach), has proven useful as a biochemical marker of cell differentiation in developmental biology. For instance, studies of the appearance of AchE during development of the vertebrate nervous system have shown that, in the fetal rat (Kupfer and Koelle, 1951) and the chick embryo (Mumenthaler and Engel, 1961), some AchE is already present in the developing cholinoceptive muscles prior to innervation of cholinergic motor neurons. However, high levels of muscle AchE arise only after innervation at the neuromuscular junctions (Vigny et al., 1976).

The presence of AchE also has been detected at much earlier developmental stages, long before the overt differentiation of any nerve or muscle tissue. For instance, AchE has been found in the uncleaved eggs of the sea urchin Pseudocentrolus depressus (Ozaki, 1974) and of the ascidian Ciona intestinalis (Meedel and Whittaker, 1979). Thus, the localization of AchE can assist in cell lineage analysis and the segregation of cytoplasmic factors in development (Whittaker, 1973). Precursor cells of

\footnotetext{
1 This work was supported by National Institutes of Health Research Grant NS 12818 and National Science Foundation Research Grant BNS79-12400. We thank Jeffrey Winer, David Weisblat, Duncan Stuart, and Andrew Kramer for advice and suggestions and Sun Yung Kim for technical assistance.

${ }^{2}$ To whom correspondence should be addressed at her current address: Department of Biology, Leidy Laboratories, University of Pennsylvania, Philadelphia, PA 19104.
}

muscle tissue in Ciona exhibit localized AchE activity as early as the eight-cell stage. However, these precursor cells give rise to larval rather than adult tissue. Localization of AchE in the nervous system occurs only after the postmetamorphic zooid stage is reached.

This report presents the results of a study of the presence of AchE during embryonic development of a glossiphoniid leech. In the adult hirudiniid leech Hirudo medicinalis, AchE is found in the muscles of the anterior sucker, in the longitudinal and dorsoventral muscles, and in the neuropil of the segmental ganglia of the ventral nerve cord (Silver, 1974). Accordingly, the AchE inhibitor eserine enhances the action of Ach on the longitudinal muscles of Hirudo (Flacke and Yeoh, 1968). Since preliminary observations indicated that a similar distribution of AchE occurs also in the adult nerve and muscle tissue of the embryologically more accessible glossiphoniid leech Helobdella triserialis, embryos of Helobdella were examined for their content and localization of AchE at various developmental stages.

Glossiphoniid leeches lay large, yolk-rich eggs that undergo a series of stereotyped cleavages. These cleavages result in formation of a set of blastomeres that are individually identifiable on the basis of their size, shape, and location within the embryo. Each blastomere gives rise to some specific adult tissue without formation of any transient larval structures (Whitman, 1878, 1887). Three of the blastomeres (A, B, and C), present at the four-cell stage, give rise to endoderm and the fourth (D) 

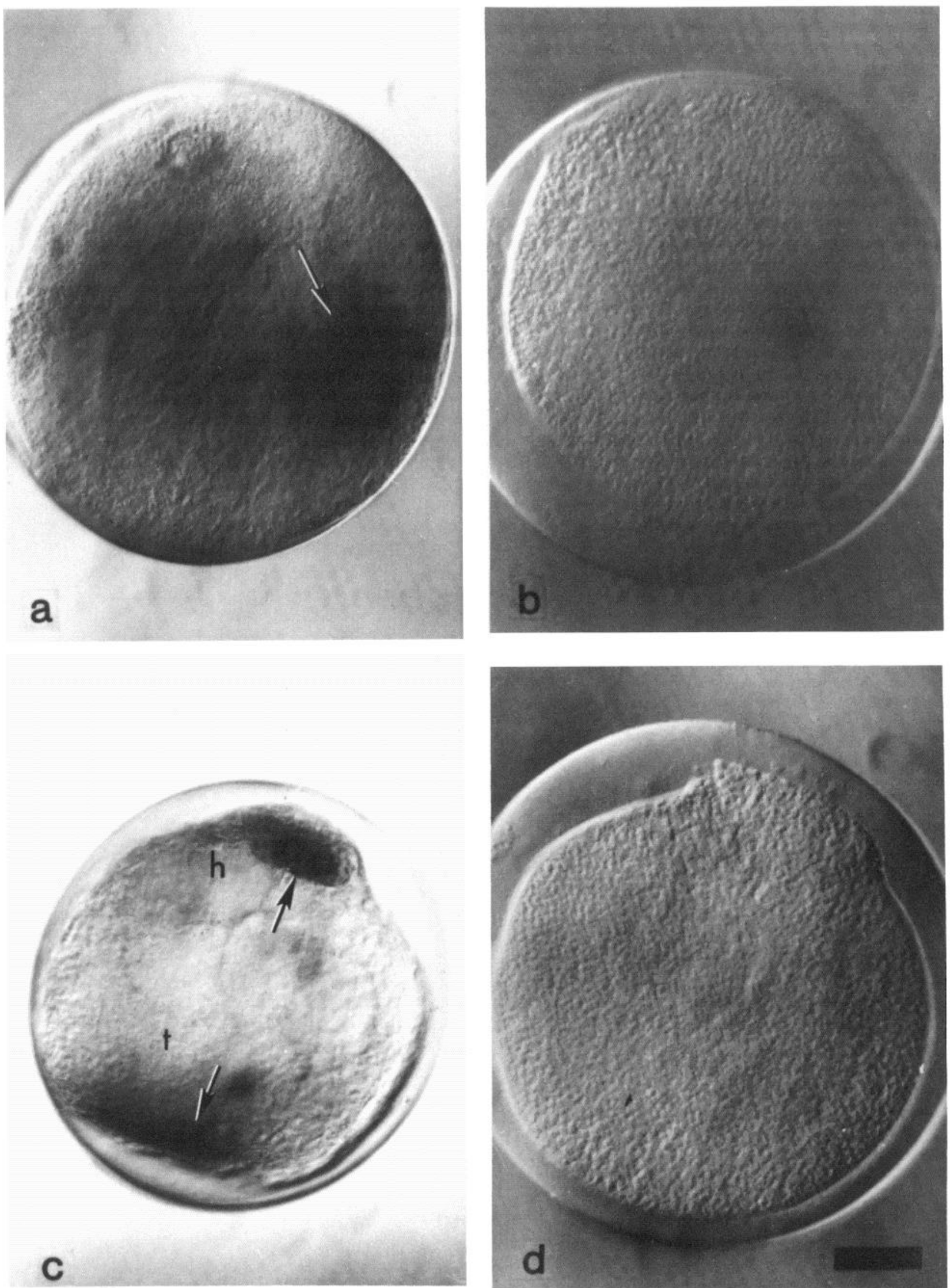

Figure 1. Specificity of the AchE staining in Helobdella embryos. a, An uncleaved egg shortly after laying (early stage 1) stained for cholinesterase. The arrow points to a diffuse band of dark stain. $b$, An egg similarly treated in the presence of eserine. $c$, Dorsal view of a stage 8 embryo stained for cholinesterase. Dark staining can be seen at the future head $(h)$ and tail $(t)$ regions of the germinal plate. $d$, A stage 8 embryo similarly treated in the presence of eserine. The scale bar represents $100 \mu \mathrm{m}$ for $a, b$, and $d$ and $110 \mu \mathrm{m}$ for $c$. 
gives rise to mesoderm and ectoderm. Subsequent cleavage of blastomere $D$ results in one bilateral pair of mesoteloblasts (M) and four bilateral pairs of ectoteloblasts $(\mathrm{N}, \mathrm{O}, \mathrm{P}$, and $\mathrm{Q})$. Of the ectoteloblasts, the $\mathrm{N}$ pair gives rise mainly (or possibly exclusively) to neural tissue, whereas the $\mathrm{O}, \mathrm{P}$, and $\mathrm{Q}$ pairs give rise to both neural tissue and non-neural ectoderm (Weisblat et al., 1978, 1980a, b).

The early phase of embryogenesis of Helobdella, from uncleaved egg to completion of the germinal plate, has been subdivided into eight stages (Weisblat et al., 1980a). During that early phase, the embryo is enclosed in a clear vitelline membrane and has a diameter of about $500 \mu \mathrm{m}$. The first eight stages, which extend over about 5 days (at $25^{\circ} \mathrm{C}$ ), include blastomere cleavage and formation of the germinal bands. They culminate in formation of the germinal plate by coalescence of the germinal bands at the future ventral midline. These stages are followed by the late phase of embryogenesis, when the germinal plate continues to expand laterally and differentiates into the segmental organ systems. Two to 3 days after the completion of stage 8 , right and left lateral edges of the cxpanding germinal plate have met and joined at the dorsal midline to close the leech body. By the time of body closure, the proboscis and caudal sucker have been formed. Moreover, at this time, the embryo has a ventral nerve cord whose gross anatomy resembles that of the adult leech with respect to the number of segmental ganglia and the presence of neuropil, connectives, and segmental nerves (Fcrnandez, 1980). About 4 days after body closure, the late phase of embryogenesis culminates in the formation of the juvenile leech, whose general morphology resembles that of the adult leech.

The work reported here shows that AchE has appeared in the musculature and ganglionic neuropil by the time of body closure of the leech embryo. Moreover, primordial AchE is already present in the uncleaved leech egg and during the early phase of leech embryogenesis when, prior to tissue formation, AchE is localized in the teloblasts, the germinal bands, and the germinal plate.

\section{Materials and Methods}

Experiments were carried out with embryos of the small (1-cm-long) glossiphoniid leech Helobdella triserialis obtained from a breeding colony maintained in this laboratory. The methods of removing Helobdella embryos from their cocoons, which are attached to the parental venter, and of culturing them in saline, as well as the criteria for identifying successive developmental stages, have been described by Weisblat et al. (1980a).

The presence of acetylcholinesterase (AchE) was detected by the histochemical method of Karnovsky and Roots (1964), using acetylthiocholine as the AchE substrate. This method produces a brown precipitate of thiocholine and potassium ferricyanide localized at the sites of AchE activity. Moreover, the density of the precipitate provides a means of quantifying the amount of AchE present at the site. Prior to staining for AchE, early embryos at stages 1 to 8 were rendered transparent by the acid-clearing method of Fernandez (1980). Late embryos at the body closure stage were pre-fixed in acidified $80 \%$ ethanol for $3 \mathrm{~min}$ at $8^{\circ} \mathrm{C}$ prior to $\mathrm{Ach}$ staining (Meedel and Whittaker, 1979). Embryos of each stage examined were divided into an experimental and a control group. Embryos of the experimental group were incubated in the staining reaction mixture for $16 \mathrm{hr}$ at $8^{\circ} \mathrm{C}$. Embryos of the control group were incubated in the staining reaction mixture in the presence of $10^{-4} \mathrm{M}$ eserine, which selectively inhibits AchE and butyrylcholinesterase (BuchE) but allows expression of nonspecific esterases (Koelle, 1950). The embryonic cholinesterase was identified as AchE rather than BuchE by the use of two selective inhibitors at a concentration of $10^{-4} \mathrm{M}$ : tetramonoisopropyl pyrophosphortetramide (isoOMPA), an inhibitor of pseudocholinesterase that allows the expression of AchE, and 1:5-bis(4-allyldimethyl ammonium phenyl)pentan-3-onedibromide (BW284 C51), an inhibitor of AchE that allows the expression of BuchE. All embryos were post-fixed in $2.5 \%$ glutaraldehyde solution as described by Weisblat et al. (1980a) except that the fixative also contained $0.01 \%$ acetic acid to provide for additional clearing. The fixed embryos were mounted in $50 \%$ glycerol and were observed and photographed as whole mounts under differential interference contrast optics. In addition, embryos stained at the body closure stage were embedded in glycol methacrylate resin and sectioned by means of a Sorvall JB-4 microtome. From 30 to 60 embryos per stage were stained for AchE at each of 10 developmental stages in at least three separate experiments. In addition, at every stage, about 25 control embryos were tested for the specificity of the staining reaction.

\section{Results}

Figure $1 a$ shows an uncleaved Helobdella egg shortly after laying (early stage 1) which was stained for cholinesterase. A diffuse band of dark stain is present. By contrast, a control egg, similarly treated in the presence of eserine, shows no staining (Fig. 1b). Hence, it can be concluded that the stain developed in the preparation shown in Figure $1 a$ is attributable to the presence in the leech egg of cholinesterase rather than to the presence of nonspecific esterases or to non-enzymatic staining. Figure $1 c$ shows a dorsal view of the germinal plate of a more advanced Helobdella embryo (stage 8), stained for cholinesterase. Patches of intense stain are present at both the rostral and caudal extremities of the germinal plate. A stage 8 control embryo, similarly treated in the presence of eserine, shows no staining (Fig. 1d). Thus, the stain developed in the stage 8 embryo of Figure $1 c$ is similarly attributable to the presence of cholinesterase in the germinal plate. In additional experiments, the observed cholinesterase was identified as AchE. For that purpose, embryos at various stages were stained in the presence of the BuchE inhibitor iso-OMPA or of the AchE inhibitor BW284 C51. Under the former condition, full staining was observed, whereas, under the latter condition, no cellular staining was observed, although some stain was found in the vitelline membrane of the embryos.

Figure $2 a$ shows an egg just prior to cleavage (late stage 1). By this time, two regions of clear cytoplasm, the polar plasms, have separated from the yolk. These two polar plasms are composed of cytoplasmic organelles and are relatively free of yolk platelets (Fernandez, 1980; Fernandez and Stent, 1980). Both polar plasms are 

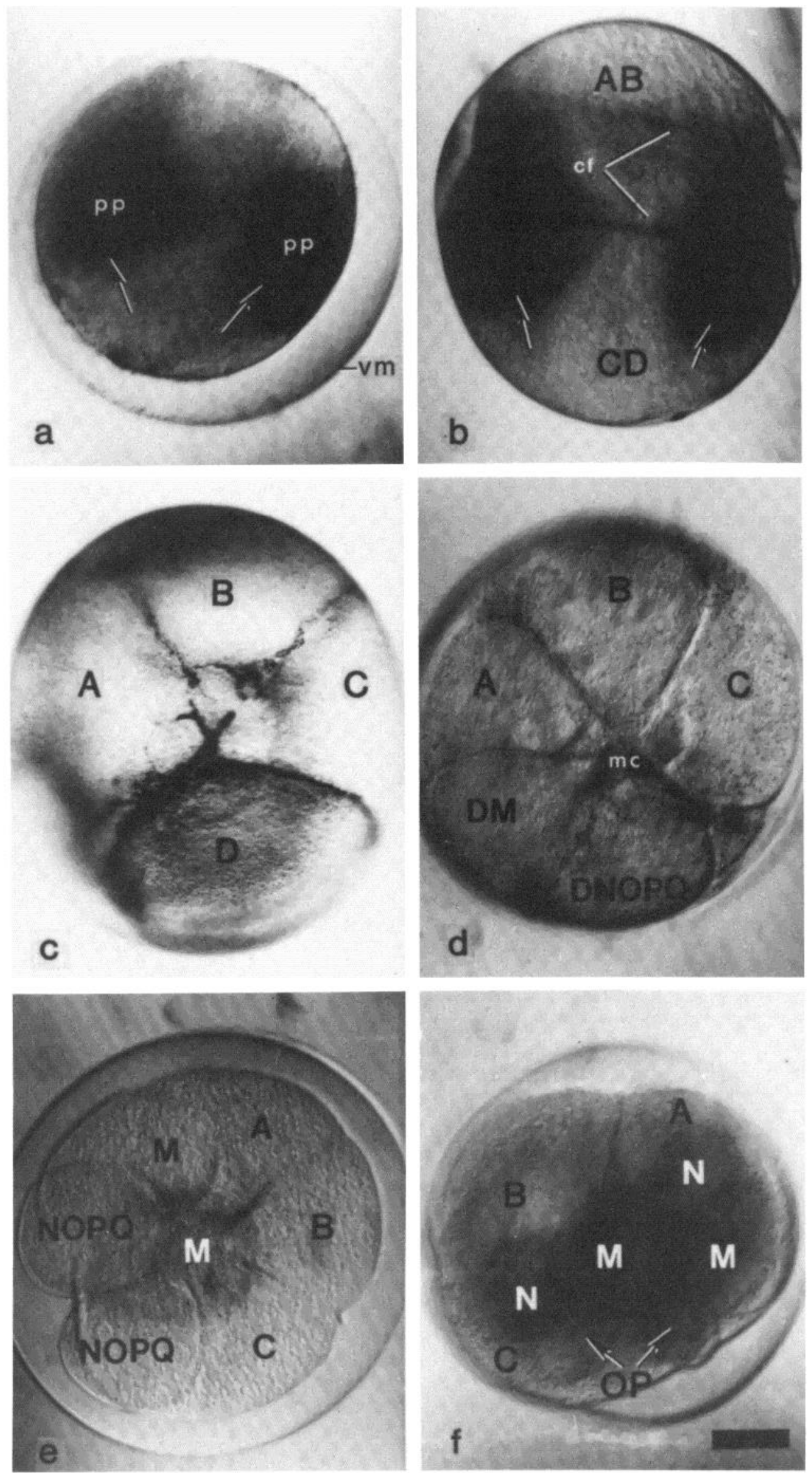
stained intensely and thus contain AchE. The difference between the diffuse band of AchE stain seen in the early stage 1 embryo of Figure $1 a$ and the two localized sectors of stain seen in the late stage 1 embryo of Figure $2 a$ evidently reflects the emergence of the polar plasms prior to the onset of the first cleavage. Upon the first cleavage of the egg into blastomeres $\mathrm{AB}$ and $\mathrm{CD}$ (stage 2), the bulk of the polar plasms is transmitted to $\mathrm{CD}$. In accord with this fact, it can be seen in Figure $2 b$ that also the bulk of the two cytoplasmic sectors staining for AchE is passed on to $\mathrm{CD}$ rather than to $\mathrm{AB}$. Moreover, a high density of AchE stain lines the cleavage furrow separating $\mathrm{AB}$ and $\mathrm{CD}$ (Fig. $2 b$ ). At the four-cell stage (stage 3), most of the AchE is present in blastomere $D$ (which is known to receive also the bulk of the polar plasm). Very little AchE stain is present in blastomeres A, B, and C. It should be noted that the cleavage furrows surrounding blastomere $\mathrm{D}$ stained more densely than other furrows (Fig. 2c).

The capacity of the embryo for AchE staining diminishes during formation of the micromere cap and the cleavage of blastomere D to yield the ecto- and mesoteloblast precursors DNOPQ and DM. Although this diminished stainability was obvious on sight, microdensitometer readings of stain intensity on color slides taken under bright-field illumination substantiated the gradual diminution of the capacity of the embryo to take on AchE stain. In dorsal views of the embryo at stage 4, light stain is manifest in the ectoteloblast precursor DNOPQ, in the mesoteloblast precursor DM, and in the micromere cap (Fig. 2d). During the next two stages (stages 5 and 6), which culminate in the formation of the one pair of mesoteloblasts and the four pairs of ectoteloblasts, most embryos stain only weakly on the surface of their teloblasts (Fig. 2, $e$ and $f$ ). Similar decreases in staining capacity were observed also in frozen sections of embryos at these developmental stages.

The capacity of the embryo to stain for AchE returns upon the onset of germinal band formation. During stage 7 , each teloblast produces a column or bandlet of stem cells which eventually merges with the other four ipsilateral bandlets to form the germinal bands. At this stage, the teloblasts and stem cell bandlets stain for AchE, but blastomeres A, B, and C do not (Fig. 3a). Examination of the bandlets at higher magnification reveals that their stain is concentrated mainly at the contact surfaces between stem cells. Upon completion of the early phase of embryogenesis (stage 8), the germinal plate, as shown in the lateral view of an embryo in Figure $3 b$, stains heavily for AchE. As can be seen in the dorsal view of a similar stage 8 embryo in Figure 1c, the AchE stain forms a ring at the rostral end and a flat band at the caudal end of the germinal plate.
In late embryogenesis, by the time of body closure of the Helobdella embryo, AchE is present in the ventral nerve cord, in the body wall, and in the distal part of the proboscis (Fig. 3c). Within the neuropil of each segmental ganglion of the embryonic nerve cord, the AchE stain forms a bilateral pair of longitudinal bands as well as some commissural tracts (Fig. 3d). By contrast, the nerve cell bodies (located in the ganglionic cortex) and the interganglionic nerve tracts are stained very lightly for AchE. The neuropils of the caudal ganglion and of the subesophageal ganglion (Fig. 3c) are stained darkly, whereas the supraesophageal ganglion is stained lightly for AchE. In the body wall, the AchE stain forms circumferential streaks (Fig. 3c). These streaks are likely to represent $\mathrm{AchE}$ present at circumferentially aligned sites of motor neuron innervation of the body wall musculature, especially of the longitudinal muscle fibers. At the distal end of the proboscis, the AchE stain forms a ring, probably representing aligned sites of innervation on the proboscis muscle (Fig. 3c). The darkly stained remnants of the teloblasts can be seen in the gut.

\section{Discussion}

The present finding of AchE in the polar plasm and cleavage furrows of early leech embryos lends support to Buznikov's (1971) suggestion (based on studies with sea urchin embryos) that neurotransmitters, such as Ach, play some role in embryogenesis. Indeed, as the discovery by Kusano et al. (1977) of Ach receptors in the frog oocyte cell membrane suggests, this role may even begin in oogenesis. However, just what that role is remains unknown. The preferential transmission of the AchE initially present in the uncleaved leech egg to the CD blastomere and then to the D blastomere is fully consonant with the previously ascertained mode of preferential transmission to cell $\mathrm{D}$ of the polar plasm (Fernandez, 1980; Fernandez and Stent, 1980). The decrease in AchE activity during early embryogenesis, and the near disappearance of the AchE from the embryo by stage 6 , could be attributable simply to the gradual intracellular degradation of the AchE originally acquired during oogenesis. In that case, the reappearance of AchE activity at stage 7 in teloblasts and their daughter stem cells might reflect the onset of gene-directed protein synthesis in the embryo in accord with the observation that, at that same stage, prominent nucleoli are seen first in the embryonic leech nuclei (Fernandez and Stent, 1980). This finding of a reappearance of AchE in the stage 7 embryo resembles the observation of Meedel and Whittaker (1979) of a rapid increase of AchE activity in the Ciona embryo at gastrulation, the time at which it generally has been found that embryos first synthesize proteins encoded in their own genome (Davidson, 1976). In the stage $7 \mathrm{em}$ -

Figure 2. Helobdella embryos of stages 1 through 6 stained for AchE. a, Egg just prior to first cleavage (late stage 1). The two polar plasms $(p p)$ are stained darkly (arrows). The egg is surrounded by the vitelline membrane (vm). $b$, The embryo after the first cleavage (stage 2). The stainable polar plasms (arrows) have been passed on to the larger CD blastomere. The cleavage furrow ( $f$ ) separating blastomeres $\mathrm{AB}$ and $\mathrm{CD}$ is seen as a ring of stain. $c$, Stage 3 embryo. Stain is present mainly in blastomere $\mathrm{D}$ and its surrounding cleavage furrows. $d$, Stage 4 embryo. The mesoteloblast precursor DM, the ectoteloblast precursor DNOPQ, and the micromere cap $(m c)$ are stained lightly. $e$, Stage 5 embryo. Very little stain is present in the $M$ mesoteloblast pair or in the NOPQ ectoteloblast precursor pair. $f$, Stage 6 embryo (ventral view). The M mesoteloblast pair, the $N$ ectoteloblast pair, and the OP ectoteloblast precursor pair are stained lightly. The scale bar represents $120 \mu \mathrm{m}$ for $a$ and $100 \mu \mathrm{m}$ for $b$ through $f$. 

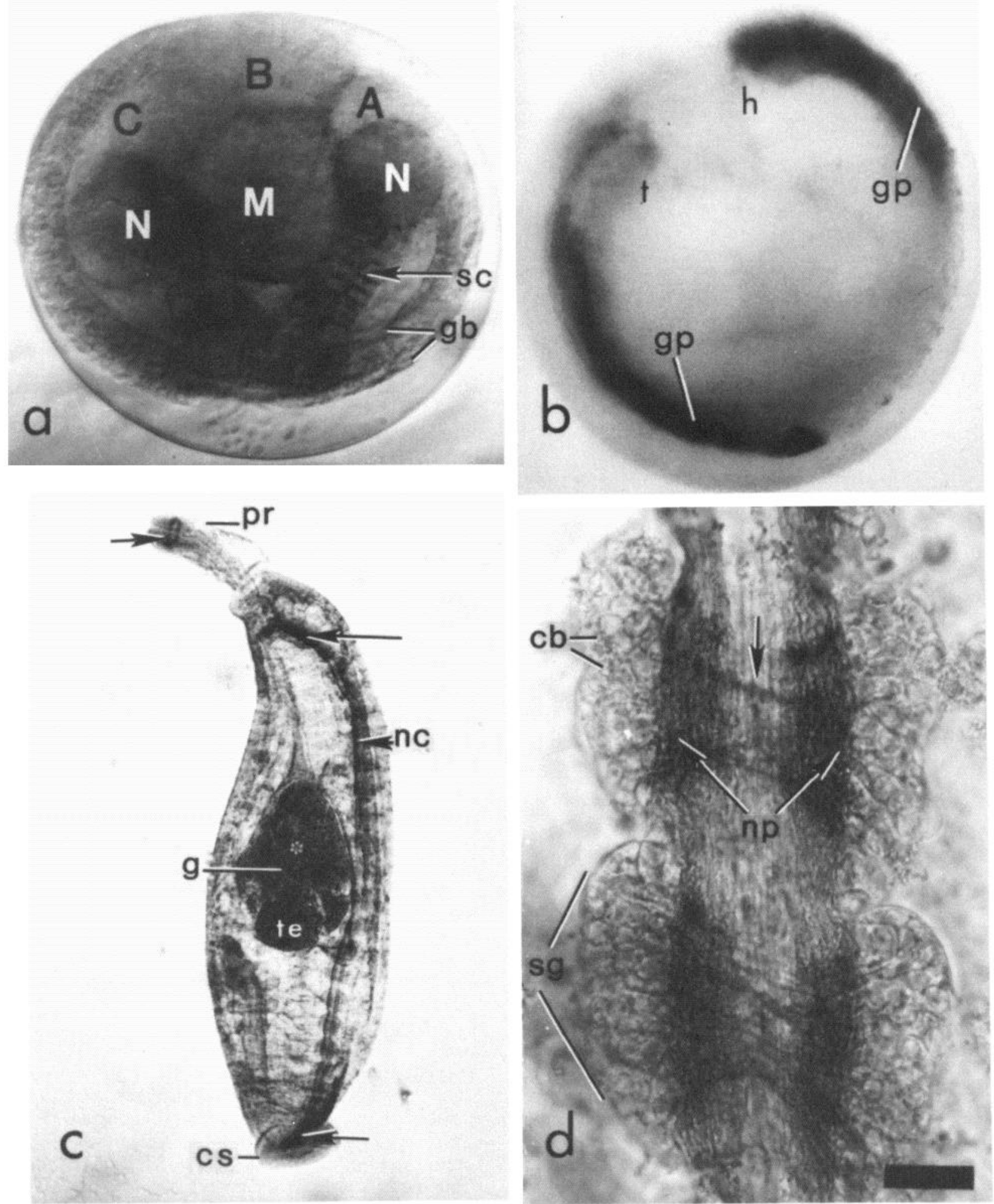

Figure 3. Helobdella embryos of later developmental stages stained for AchE. a, Stage 7 embryo (ventral view). AchE is localized in the $\mathrm{M}$ and $\mathrm{N}$ teloblasts and in their descendant stem cells $(s c)$ which form the germinal bands $(g b)$. $b$, Stage 8 embryo (lateral view). AchE is present in the germinal plate $(g p)$, extending clockwise from the future head $(h)$ to the future tail $(t)$. $c$, Body closure stage. Arrows point to the AchE present in the neuropil of the ventral nerve cord (nc) in the presumptive neuromuscular contacts of proboscis $(\mathrm{pr})$ and caudal sucker $(\mathrm{cs})$. The asterisk marks the stain attributable to teloblast remnants $(t e)$ present in the gut $(g) . d$, Longitudinal section of two segmental ganglia $(\mathrm{sg})$ of a preparation similar to that shown in $c$. Arrows point to the AchE present in the ganglionic neuropil $(n p)$ and in some commissures connecting the two sides of each ganglion. The central longitudinal nerve tract containing interganglionic axons and the nerve cell bodies $(c b)$ are only stained lightly. The scale bar represents $100 \mu \mathrm{m}$ for $a$ and $b, 180 \mu \mathrm{m}$ for $c$, and $15 \mu \mathrm{m}$ for $d$. 
bryo, there is as yet no neurological function for neurotransmitters, since no nervous system has yet been formed. Thus, it seems possible that AchE (and Ach) plays some role in the development of the germinal bands.

The presence of AchE in the neuropil of the segmental ganglia and in the musculature at the time of body closure in late embryogenesis indicates that, at this still quite immature developmental stage, the embryonic nervous system has taken on not only the morphological but also some of the neurochemical characteristics of the adult leech. That the supraesophageal ganglion stains much less intensely for AchE than do all other ganglia of the ventral nerve cord supports the previous inference that the supraesophageal ganglion has a developmental origin which is different from that of the ventral nerve cord, namely blastomeres $\mathrm{A}, \mathrm{B}$, or $\mathrm{C}$, rather than the meso- and ectodermal precursor blastomere D (Weisblat et al., 1980a).

\section{References}

Buznikov, G. A. (1971) The role of nervous system mediators in individual development. Ontogenez 2: 5-13.

Davidson, E. H. (1976) Gene Activity in Early Development, Ed. 2, Academic Press, New York.

Fernandez, J. (1980) Embryonic development of the glossiphoniid leech Theromyzon rude: Characterization of developmental stages. Dev. Biol. 76: 245-262.

Fernandez, J., and G. S. Stent (1980) Embryonic development of the glossiphoniid leech Theromyzon rude: Structure and development of the germinal bands. Dev. Biol. 78: 407-434.

Flacke, W., and T. S. Yeoh (1968) The action of some cholinergic agonists and anticholinesterase agents on the dorsal muscle of the leech. Br. J. Pharmacol. 33: 145-153.

Karnovsky, M. J., and L. Roots (1964) A "direct-coloring" thiocholine method for cholinesterases. J. Histochem. Cytochem. 12: 219-221.

Koelle, G. B. (1950) The histochemical differentiation of types of cholinesterase and their localizations in tissues of the cat. J. Pharmarol. Fxp. Ther. 100: 158-191.

Kupfer, C., and G. B. Koelle (1951) A histochemical study of cholinesterase during formation of the motor endplate of the albino rat. J. Exp. Zool. 116: 397-413.

Kusano, K., R. Miledi, and J. Stinnakre (1977) Acetylcholine receptors in the oocyte membrane. Nature 270:739-741.

Meedel, T. H., and J. R. Whittaker (1979) Development of acetylcholinesterase during embryogenesis of the ascidian Ciona intestinalis. J. Exp. Zool. 210: 1-10.

Mumenthaler, M., and W. K. Engel (1961) Cytological localization of cholinesterase in developing chick embryo skeletal muscle. Acta Anat. (Basel) 47: 274-299.

Ozaki, H. (1974) Localization and multiple forms of acetylcholinesterase in sea urchin embryos. Dev. Growth Differ. 16: 267-279.

Silver, A. E. (1974) Subcellular localization, synthesis and trans. port of acetylcholinesterase. In Frontiers of Biology, A. Neuberger and E. L. Tatum, eds., pp. 99-131, American Elsevier, New York

Vigny, M., J. Koenig, and F. Rieger (1976) The motor end-plate specific form of acetylcholinesterase: Appearance during embryogenesis and reinnervation of rat muscle. J. Neurochem. 27: 1347-1353.

Weisblat, D. A., R. T. Sawyer, and G. S. Stent (1978) Cell lineage analysis by intracellular injection of a tracer enzyme. Science 202: 1295-1298.

Weisblat, D. A., G. Harper, G. S. Stent, and R. 'T. Sawyer (1980a) Embryonic cell lineages in the nervous system of the glossiphoniid leech Helobdella triserialis. Dev. Biol. 76: 5878.

Weisblat, D. A., S. L. Zackson, S. S. Blair, and J. D. Young (1980b) Cell lineage analysis by intracellular injection of fluorescent tracers. Science 209: 1538-1541.

Whitman, C. O. (1878) The embryology of Clepsine. Q. J. Microsc. Sci. 18: 215-315.

Whitman, C. O. (1887) A contribution to the history of germ layers in Clepsine. J. Morphol. 1: 105-182.

Whittaker, J. R. (1973) Segregation during ascidian embryogenesis of egg cytoplasmic information for tissue-specific enzyme development. Proc. Natl. Acad. Sci. U. S. A. 70: 2096-2100. 